\title{
1 Modified recipe to inhibit GSK-3 for the living fungal
}

\section{biomaterial manufacture}

3 Jinhui Chang $^{11}$, Po Lam Chan ${ }^{19}$, Yichun Xie ${ }^{1}$, Man Kit Cheung ${ }^{1}$, Ka Lee Ma ${ }^{1}$, Hoi Shan

$4 \operatorname{Kwan}^{1 *}$

$5{ }^{1}$ School of Life Sciences, The Chinese University of Hong Kong, Shatin, New Territories,

$6 \quad$ Hong Kong

$7 \quad *$ Corresponding author: E-mail: hskwan@eservices.cuhk.edu.hk

$8 \quad$ These authors contributed equally to this work.

9

10

11 


\section{Abstract}

13 Living fungal mycelium with suppressed or abolished fruit-forming ability is a self-healing

14 substance particularly valuable biomaterial for further engineering and development in

15 applications such as monitoring/sensing environmental changes and secreting signals. The

16 ability to suppress fungal fruiting is also a useful tool for maintaining stability (e.g., shape,

17 form) of a mycelium-based biomaterial with ease and lower cost.

18 The objective of this present study is to provide a biochemical solution to regulate the fruiting

19 body formation to replace heat killing of mycelium during production. We discovered that

20 GSK-3 activity directly correlates with the development of fruiting bodies in fungi, especially

21 mushroom forming fungi such as Coprinopsis cinerea. By regulating GSK-3 expression and

22 activity, one can control the fungal fruiting body development.

23 We successfully demonstrated that treatment of an inhibitor of GSK-3 kinase activity resulted

24 in acceleration in mycelium growth rate, absence of fruiting body and general decrease in

25 GSK-3 gene expression. Therefore, GSK-3 inhibitor is suggested to be included in the mycelium cultivation recipes for regulating the growth of fungal mycelium and for inhibiting

27 the development of fruiting bodies. This is the first report of using a GSK-3 inhibitor, such as

28 lithium or any other GSK-3 inhibitor, to suppress or abolish fruiting body formation in living

29 fungal mycelium-based biomaterial. It also provides an innovative strategy for easy, reliable, and low cost maintenance of biomaterial containing live fungal mycelium. 


\section{Introduction}

34 Fungal mycelium-based biomaterials are fast emerging in recent years. Mycelium is the vegetative structure of fungi, mainly composed of natural polymers. Mycelium-based biomaterials have a wide range of applications due to their controlled and tunable properties during growth, its self-assembly, self-healing, environmentally responsive, and biodegradable nature. The dried mycelium has strength, durability, and many other beneficial qualities: it is nontoxic, fire-resistant, mold resistant, water-resistant, and a great thermal insulator, amongst other salient features(1-13). Under proper circumstances, mycelium of many mushroomforming fungi will aggregate to form mushrooms, which are the fruiting body spreading spores(14). Not only the fruiting bodies will cause conformational changes of the mycelium based materials, but also the spores may cause allergy and infection in susceptible population. In current production procedures of the mycelium-based materials, whole material are heated or treated with fungicide to kill the living cells, to stop the fruiting body formation(15). Such rendered mycelium-based materials retain few of the benefits of living material. Therefore, new approaches are needed for inhibiting fruiting body formation while keeping the mycelium alive, in order to produce living mycelium-based materials of desirable qualities.

Kinases mediate cellular and developmental responses to growth factors, environmental signals, and internal processes, and the kinases cascades play crucial roles in many signaling transduction pathways $(16,17)$. Phosphorylation of proteins kinases affects their activity, localization, stability, conformation, and protein-protein interaction. One interesting and putatively central regulatory kinase is glycogen synthase kinase-3 (GSK-3). GSK-3 is a serine/ threonine kinase of the CMGC family of proline-directed kinases that is highly conserved in all eukaryotes. GSK-3 is activated by the constitutive phosphorylation at a Cterminal tyrosine residue, however, the regulatory phosphorylation at an $\mathrm{N}$-terminal serine 
residue causes a conformational change to block the catalytic domain, hence inhibits its kinase activity (18). The kinases PKA, PKB, and PKC inhibit GSK-3 in specific signaling pathways in eukaryotes, while in fungi these kinases are essential growth regulators in

60 response to environmental stimuli(19-21).

61 In mammals, GSK-3 inhibition has attracted widespread attention as one of the critical

62 therapeutic targets whereby lithium exerts its pharmacological effects on mood stabilization, 63 neurogenesis, neurotrophicity, neuroprotection, anti-inflammation, and others(18). Lithium compounds are also suggested to be added in cultivation to fortify the lithium nutrient value of some edible mushrooms $(22,23)$. Lithium chloride $(\mathrm{LiCl})$ is a well-known substance that has been shown to inhibit GSK-3 and recent evidence suggests that low, non-toxic concentrations of such a compound have indeed anti-inflammatory effects(24).

In this study, Coprinopsis cinerea is used to represent the white-rot basidiomycetous fungi, as

69 it is a classic model mushroom-forming fungus(25). The typical life cycle of $C$. cinerea can be finished within 2 weeks under lab condition, which includes stages of basidiospores, vegetative mycelium, hyphal knots, initial, stage-1 and -2 primordia, young and mature fruiting bodies(26).

This study aims to provide a biochemical approach to inhibit fruiting body formation from the mycelium-based biomaterals, thus producing living biomaterials. We demonstrated that fruiting body development in mushrooms can be regulated by modulating GSK-3 expression and/or activity: suppression of GSK-3 expression and/or activity can promote the growth of mycelium and inhibit the fruiting body formation, whereas enhancement of GSK-3 expression and/or activity can achieve opposite effects. Regulation of GSK-3 can be applied in the manufacturing of mycelium-based biomaterials, which can shorten the production 
cycle, reduce the cost for maintenance of mycelium materials, and therefore achieve a higher

81 level of cost-effectiveness.

\section{Materials and Methods}

\section{Strains and cultivation conditions}

84 Two GSK3 inhibitors ( $\mathrm{LiCl}$ and CHIR99021-HCl) and one GSK3 activator (Cisplatin) were

85 tested in Coprinopsis cinerea, the homokaryotic fruiting strain \#326 (A43mut B43mut pab1-

1). One GSK3 inhibitor ( $\mathrm{LiCl})$ was tested in Pleurotus djamor (commonly known as the pink oyster mushroom). Belonging to the same order Agaricales, these two tested mushroom species are of two different families, Psathyrellaceae and Pleurotaceae, respectively. $C$. cinerea is cultured on yeast extract-malt extract-glucose (YMG) agar plates (per litre, $4 \mathrm{~g}$ yeast extract; $10 \mathrm{~g}$ malt extract; $4 \mathrm{~g}$ glucose; $10 \mathrm{~g}$ agar;) at $37^{\circ} \mathrm{C}$ in the dark until mycelia grow over the whole agar surface (27). Fruiting body development is induced by incubating the mycelia at $25^{\circ} \mathrm{C}$ under a 12 hours light /12hours dark cycle. P. djamor is cultivated on Potato Dextrose Agar (PDA, BD Difco ${ }^{\mathrm{TM}}$ ) plates at $28^{\circ} \mathrm{C}$ in the dark until mycelia grow over

94 the whole agar surface, and transferred to $25^{\circ} \mathrm{C} 12$ hours light /12hours dark cycle to induce fruiting body formation. Triplicates were employed in each set up. Each plate was measured for $45 \mathrm{~g}( \pm 1 \mathrm{~g})$ medium to uniform the nutrients contents and for accurate inhibitor

97 concentration.

\section{Effect of Inhibitors and Activator}

Inhibitors of GSK-3 used in this study include lithium chloride ( $\mathrm{LiCl})$, as well as CHIR99021

100 trihydrochloride. Three methods have been tested to deliver LiCl (Sigma-Aldrich, St. Louis, 
autoclave sterilization, and the other methods are to spread $\mathrm{LiCl}$ solution on the surface of agar before inoculation, or add $\mathrm{LiCl}$ solution under the agar after mycelia reaches the petri dish edge. CHIR99021 trihydrochloride is very specific and water soluble, so it is used to confirm the effect of inhibited GSK-3 on fruiting body development. 5nM, 10nM and 15nM of CHIR99021 trihydrochloride is spread on the surface of agar evenly before inoculation.

107 In this study, one specific GSK-3 activator, cisplatin, is tested. It is also known as cisplatinum, platamin, neoplatin, cismaplat, or cis-diamminedichloridoplatinum(II) (CDDP), a chemical most commonly used in chemotherapy in cancer treatment. $1 \mathrm{ml}$ Water or saturated Cisplatin solution $\left(25^{\circ} \mathrm{C}\right)$ was filtered and spread on the surface of YMG agar,

111 before inoculation. CHIR99021 trihydrochloride and cisplatin are not suggested to be 112 autoclaved, so we use 0.2 micron filter to remove all bacteria in the solution.

\section{Sensitive window to $\mathbf{L i C l}$}

114 The effect of $\mathrm{LiCl}$ at different developmental stages of $C$. cinerea are tested to find the

115 sensitive window. $0.1 \mathrm{ml}$ of $3 \mathrm{~g} / \mathrm{L} \mathrm{LiCl}$ or $0.1 \mathrm{ml}$ water were added under the agar at the 116 stages of: mycelium, hyphal knot, initial, stage-1 primordium, stage-2 primordium and young

117 fruiting body. The growth status was record till 3 days after the control group form mature 118 fruiting bodies.

\section{Expression levels of GSK-3 downstream target genes}

120 The expression levels of targets genes of GSK-3 were measured to explore the mechanism of

121 LiCl. The GSK-3 downstream targets were predicted by Orthologue comparison. A total of

12283 GSK-3 downstream targets reported in human and mouse, were compared to the $C$. 
124 glycogen synthase (CC1G_01973), GSK-3 (CC1G_03802), eukaryotic translation initiation

125 factor 1 (eIF1) (CC1G_03881), uncharacterized protein with Ricin B-type lectin domain

126 (CC1G_05298), translation initiation factor eIF2 gamma subunit (CC1G_09429) were picked

127 for real-time PCR analysis. For each gene, primers for two segments with similar PCR

128 condition were selected.

129 Samples treated by water or $1.5 \mathrm{~g} / \mathrm{L}$ or $3 \mathrm{~g} / \mathrm{L} \mathrm{LiCl}$ (mixed before autoclave) were cultured as

130 conditions described above. Once the control group started fruiting body initiation, total RNAs

131 of three biological replicates were extracted using RNeasy® Plant Mini Kit (Qiagen). RNA

132 products were stored at $-80^{\circ} \mathrm{C}$. The concentration of RNA was measured by NanoDrop

133 Spectrophotometers (Thermo Scientific). 500ng RNA products were used to synthesize cDNA

134 using iScript TM cDNA Synthesis Kit (Bio-Rad). Quantitative real-time PCR (qPCR) was

135 performed by Applied Biosystems ${ }^{\circledR}$ 7500/7500 Fast Real-Time PCR system ${ }^{\mathrm{TM}}$ (Applied

136 Biosystems) using iQTM SYBR ${ }^{\circledR}$ Green Supermix (Bio-Rad). 


\section{Results}

\section{Effect of GSK-3 inhibitors and activator on fruiting body}

\section{9 development}

140 As shown in Figure 1a, effect of $\mathrm{LiCl}$ to $C$. cinerea fruiting body development was tested.

141 While the control group has already developed into mature fruiting bodies, primordium,

142 initials and hyphal knots were formed on the plates treated with $1.5 \mathrm{~g} / \mathrm{L} \mathrm{LiCl}$ respectively.

143 The plates treated with $3 \mathrm{~g} / \mathrm{L} \mathrm{LiCl}$ and $6 \mathrm{~g} / \mathrm{L} \mathrm{LiCl}$ were arrested in mycelium stage, and

144 mycelium treated with $6 \mathrm{~g} / \mathrm{L} \mathrm{LiCl}$ grew than other groups. These results showed that $\mathrm{LiCl}$ of

145 higher concentrations have stronger inhibitory effect on $C$. cinerea fruiting body

146 development.

147 The three delivery methods of LiCl, either mixed in the agar, on the surface of agar, or under

148 the agar, show no differences and all can efficiently inhibit the fruiting body development.

$149 \mathrm{LiCl}$ is not sensitive to heat treatment, and can be autoclaved to sterilize the solution. Any of

150 these delivery methods can be chosen in a large-scale manufacture of the biomaterials.

151 In order to confirm that the inhibition of fruiting body development is caused by GSK-3

152 inhibition, another more specific GSK-3 inhibitor, CHIR99021 trihydrochloride, was tested.

153 As shown in $1 \mathrm{~b}$,Young fruiting bodies developed on the control plates treated with water and

154 the plates with $1 \mu \mathrm{M}$ CHIR99021 trihydrochloride. The plates treated with $100 \mu \mathrm{M}$

155 CHIR99021 trihydrochloride developed primordium. The plates treated with $500 \mu \mathrm{M}$

156 CHIR99021 trihydrochloride remained arrested in mycelium stage. These results showed an

157 stronger inhibitory effect on $C$. cinerea fruiting body development by CHIR99021

158 trihydrochloride at higher concentrations. 
$159 \mathrm{LiCl}$ and CHIR99021 trihydrochloride treatments inhibited the fruiting body initiation in a

160 dose-dependent manner (Figure 1a and 1b). Given that both $\mathrm{LiCl}$ and CHIR99021

161 trihydrochloride are specific inhibitors to GSK-3, it can be concluded that the effect of

162 lithium is mediated through the inhibition of GSK-3 activity.

163 To demonstrate that GSK-3 is also important in fruiting body formation in other mushrooms,

164 the effect of $\mathrm{LiCl}$ to Pleurotus djamor fruiting body development was tested (Figure 1c). C.

165 cinerea and $P$. djamor are two fungal species within the same family of Basidiomycota and

166 the same order of Agaricales.

$167 \mathrm{LiCl}$ was added to YMG agar medium before autoclave. Mature fruiting bodies developed

168 on the control plates. The plates treated with $2 \mathrm{~g} / \mathrm{L} \mathrm{LiCl}$ failed to develop fruiting body in the

169 following 30 days. These results showed an inhibitory effect on $P$. djamor fruiting body

170 development by LiCl.

171 With the positive results that GSK-3 inhibitor can inhibit the fruiting body development, it is hypothesized that GSK-3 activity is associated with the fruiting body development. Then a

173 GSK-3 activator, cisplatin, was tested for its effect on $C$. cinerea fruiting body development

174 (Figure 1d). The activator treated group with $1 \mathrm{ml}$ saturated Cisplatin, showed an accelerated

175 development since the formation of hyphal knot, and the mature fruiting body appeared 2

176 days earlier than the control group, while the control group was slower and only developed

177 into young fruiting body. These results showed a positive or promoting effect on C. cinerea

178 fruiting body development by GSK-3 activator, Cisplatin. 
development of $C$. cinerea. Mature fruiting bodies, young fruiting bodies, primordia and initials were producd in the control group, while the $1.5 \mathrm{~g} / \mathrm{L} \mathrm{LiCl}$ treated group produced only

184 initials and primordia. No initiation was observed in the groups treated with higher

185 concentration of $\mathrm{LiCl}$, in the following 30 days. (b) The different doses of GSK-3 inhibitor,

186 CHIR99021 trihydrochloride, have different levels of inhibitory effect on the development of

187 C. cinerea. Young fruiting bodies developed in the control group and $1 \mu \mathrm{M}$ treated group, while only primordium were developed on plates treated with $100 \mu \mathrm{M}$. The plates treated with $500 \mu \mathrm{M}$ remained in mycelium stage in the following 30 days. (c) GSK-3 inhibitor, LiCl, also inhibit he development of $P$. djamor. Mature fruiting bodies, young fruiting

191 bodies, primordia and initials were produced in the control group, while the $2 \mathrm{~g} / \mathrm{L} \mathrm{LiCl}$

192 remained in mycelium stage in the following 30 days. (d) GSK-3 activator, cisplatin, accelerates the development of $C$. cinerea. After 6-days incubation, the plates treated with 1 $\mathrm{ml}$ saturated Cisplatin solution had fruiting body and began autolysis, while the control group was slower and only developed into young fruiting body.

197 These data unequivocally support the conclusion that among fungus species of the division

198 Basidiomycota, especially of the order Agaricales, GSK3 inhibitors inhibit/reduce/decelerate

199 the fruiting body formation, whereas GSK3 enhancers activate/increase/accelerate the 200 fruiting body formation.

\section{GSK-3 inhibitor promotes mycelium growth}

202 The mycelial growth rate was measured for the $\mathrm{LiCl}$ treated C. cinerea. Biological triplicates

203 were cultivated on YMD at $38^{\circ} \mathrm{C}$ in darkness, treated with different doses of LiCl. The

204 mycelium area was recorded for 4 days. Figure 2 a shows the average growth area of each 
group. As the inoculum usually need time to adapt to the new environment and absorb

207 the $\mathrm{LiCl}$ treated groups spanned faster than the control group, and after 144 hours of

208 inoculation the mycelium spanned into a circle shape of $51.8 \mathrm{~cm}^{2}$ for $1.5 \mathrm{~g} / \mathrm{L}$, and $55.1 \mathrm{~cm}^{2}$ for

$2093 \mathrm{~g} / \mathrm{L}$, while $42.9 \mathrm{~cm}^{2}$ for control group. The results show that proper concentrations of $\mathrm{LiCl}$

210 can accelerate the mycelium growth.

211 This is an ideal property of GSK-3 inhibitor for the large-scale manufacture of mycelium-

212 based biomaterial. The modified recipe with addition of porper concentration of LiCl, can not

213 only inhibit the appearance of fruiting body, but also speed up the mycelium growth, and

214 hence lower the shorten the manufacture cycle and lower the cost.

\section{Effect of GSK-3 inhibitor on gene expression levels}

216 To validate the $\mathrm{LiCl}$ is targeting on GSK-3 other than other gene, we test the expression

217 levels of the GSK-3 gene and its downstream target genes. Two segments of each gene were

218 tested for higher accuracy. The real-time PCR results show that the $\mathrm{LiCl}$ could regulate the

219 expression of the GSK-3 gene itself, as well as the selected GSK-3 target genes. GSK-3,

220 glycogen synthase, and protein with Ricin B-type lectin domain showed decrease in gene expression levels with increase of $\mathrm{LiCl}$ concentration. eukaryotic translation initiation factor 1 is stable in all conditions, while translation initiation factor eIF2 gamma subunit increase to the GSK-3 and affects its downstream genes.

226 Fig. 2 (a) Mycelial growth of C. cinerea with different doses of $\mathrm{LiCl}$, biological triplicates were used for mean calculation. The growth rate of mycelium treated with $1.5 \mathrm{~g} / \mathrm{L}$ and $3 \mathrm{~g} / \mathrm{L}$ 
$\mathrm{LiCl}$ is higher than control. (b)The gene expression levels are indicated by the abundance of segments on the cDNA, by real-time PCR. The GSK-3 and its target genes change the expression level under $\mathrm{LiCl}$ treatment. 1973-1 \& 1973-2 are two segments on the cDNA of

231 glycogen synthase (CC1G_01973), and the others are segments of the following transcripts:

232 GSK-3 (CC1G_03802); eukaryotic translation initiation factor 1 (CC1G_03881);

233 Uncharacterized protein with Ricin B-type lectin domain (CC1G_05298); Translation

234 initiation factor eIF2 gamma subunit (CC1G_09429).

\section{Sensitive Window to GSK-3 inhibitor $\mathrm{LiCl}$}

236 For the current manufacture of mycelium-base biomaterial, it's difficult to avoid the

237 formation of fruiting body. Upon environmental stimuli, including nutrient depletion,

238 light/dark cycle, and cold shock, mycelia aggregate into hyphal knot, followed by fruiting

239 body initials. Initials then develop into stage-1 and -2 primordia, young and mature fruiting

240 bodies. The companies may already have fixed production line, so we want to explore all the

241 possible procedures to introduce the GSK-3 inhibitor, specifically, LiCl, which is cheaper

242 compared to other GSK-3 inhibitors.

243 In the previous sections, $\mathrm{LiCl}$ is demonstrated to arrest the mycelium. Hyphal knot is a short

244 stage which is difficult to define by naked eyes. So we only test the stages after initials. As

245 shown in Figure 3a, adding $3 \mathrm{~g} / \mathrm{L} \mathrm{LiCl}$ at stages of initial and stage-1primordium led to the

246 arrest of their development, while stage-2 primordium and young fruiting body could

247 continue to develop into mature fruiting bodies. Intervention of $\mathrm{LiCl}$ at stages of mycelium,

248 initial and stage-1 primordium resulted in arrestment in fruiting body development, so these

249 stages are sensitive windows to $\mathrm{LiCl}$. 
251 Fig. 3 (a)The development of $C$. cinerea treated with $0.1 \mathrm{ml}$ of $3 \mathrm{~g} / \mathrm{L} \mathrm{LiCl}$ at different stage.

252 Adding 3g/L LiCl at stages of initial and stage-1 primordium led to inhibition on further

253 development. Adding $3 \mathrm{~g} / \mathrm{L} \mathrm{LiCl}$ at stages of stage-2 primordium and young fruiting body

254 cannot inhibit the fruiting body development. (b) Proposed procedures of the production

255 pipeline of living mycelium-based materials, and the GSK-3 inhibitors can be added at any

256 time from procedure 1 to 7.

257

258 For producing living mycelium-based material, the basic production pipeline is designed for

259 adding GSK-3 inhibitors, particularly the lithium or lithium salt. The production pipeline can

260 be all of part of the following procedures: 1) substrates mixing and autoclave; 2) inoculation;

261 3) mycelium 1st growth; 4) molding and pressurize; 5) mycelium 2nd growth; 6) pressurize

262 (optional); 7) mycelium 3rd growth (optional); 8) air-dry to finalized product. $\mathrm{LiCl}$ or other

263 GSK-3 inhibitors can be added at any time from procedure 1) to 7), by mixing in the

264 substrate before autoclave, adding the sterilized solution on the surface or inside the medium

265 mixture before inoculation, or spraying to the mycelium after a period of growth.

\section{Discussion}

267 The study demonstrated that GSK-3 activity can determine the fruiting body development,

268 and GSK-3 inhibitor is suggested to be included into recipes to manufacture live fungal

269 mycelium that exhibits an altered and more desirable profile of fruiting body development as

270 well as compositions that contain the fungal mycelium.

271 In many instances one would prefer for a live fungal mycelium to refrain from developing

272 fruiting bodies such that the mycelium is easily maintained without concerns of loss in its

273 shape, form, or consistency. In contrast to the currently-in-use method of heat-killing fungal 
mycelium to prevent fruiting body formation, a live version of mycelium that simply does not

275 form fruiting bodies is far more desirable considering its live nature and thus healing

276 potential.

277 In other cases, promoting fruiting body development may be of interest. For instance, when

278 the intended goal is to produce and harvest as many fruiting bodies (e.g., mushrooms and

279 truffles) as possible in a defined time period, having enhanced fruiting body development is 280 beneficial.

281 Glycogen synthase kinase-3, GSK-3 has important role in cell-fate specification, leading to cell differentiation or apoptosis or development through number of signaling pathways(2932). So we propose a pathway that GSK-3 could be the links between environmental stimuli and the responsive development, and a master-switch of fruiting body formation (Figure 4).

\section{Fig. 4: Glycogen synthase kinase-3 (GSK-3) as a master-switch of fruiting body}

287 formation. GSK-3 has important role in cell-fate specification, leading to cell differentiation or apoptosis or development through number of signaling pathways. GSK-3 could be the links between environmental stimuli and the responsive development, and a master-switch of fruiting body formation. The activity of GSK-3 determines the fruiting body development.

292 For producing a live fungal mycelium with an enhanced or inhibited fruiting body

293 development profile, either a permanent means (e.g., GSK-3 knockdown or GSK-3 knockout

294 fungal strain) or a transient means (e.g., application of an activator or inhibitor of GSK-3

295 present in the medium for fungi) can be employed. While the former may be easier to 
297 modified fungal strains are tremendously more significant both in cost and in time. In

298 contrast, the latter offers the benefits of flexibility and low-cost use, when the GSK-3

299 activator or inhibitor can be readily removed at an appropriate time such that the fungus may

300 resume its normal life cycle of different phases.

301 One possibility is to reduce or abolish GSK-3 expression by genetic manipulation of the

302 fungal cells' genomic sequence encoding the GSK-3 protein or by transient or permanent

303 expression of small inhibitory RNAs. The gene encoding GSK-3 is highly conserved across

304 diverse phyla. It is a serine/threonine kinase having a monomeric structure and a size of

305 approximately 47 kilo daltons. The amino acid sequence and corresponding polynucleotide

306 coding sequence for $C$. cinerea GSK-3 are provided in GenBank Accession Numbers

307 XP_001833585 (strain okayama7\#130) and NW_003307543.1 (Genomic Sequence in strain

308 Okayama7\#130) / jgi|Copci_AmutBmut1|363162|e_gw1.29.187.1 (in strain \#326, Taxonomy

309 ID: 1132390 and Accession: PRJNA258994), respectively. The GSK-3 is highly conserved

310 in protein sequence. The homologous proteins include PIL30457.1 in Ganoderma sinense

311 ZZ0214-1, and jgi|Gansp1|158466|gm1.11165_g in Ganoderma sp. 10597 SS1 (North

312 American isolate of G. lucidum), and KDQ33621 in Pleurotus ostreatus PC15. As used

313 herein, a GSK-3 protein encompasses both $C$. cinerea GSK-3 protein and its

314 homologs/orthologs in fungal species, especially those of Basidiomycota, having at least 90\%

315 or more sequence homology to $C$. cinerea GSK-3 protein sequence and share essentially the

316 same biological or enzymatic activity.

317 A GSK-3 knockdown cell may be generated by genetic manipulation of the genomic GSK-3

318 sequence of a suitable parent cell. Methods such as sequence homology-based gene

319 disruption methods utilizing a viral vector or CRISPR system can be used for altering the

320 GSK-3 genomic sequence, for example, by insertion, deletion, or substitution, which may 
occur in the coding region of the gene or in the non-coding regions (e.g., promoter region or other regulatory regions) and which may result in complete abolition of GSK-3 expression, reduced GSK-3 expression, or unaltered expression at mRNA level but diminished GSK-3

324 protein activity.

325 Another possibility is to suppress the activity of endogenously expressed GSK-3 protein by 326 introducing a GSK-3 inhibitor into the external environment in which the fungi grow.

327 Lithium salts have mycelium-enhancing effect to some mushroom forming fungi, but the concentration range of such effect is narrow. In some other mushroom forming fungi, high concentration of $\mathrm{LiCl}$ may inhibit the mycelium growth, especially in Trichoderma species, which is a common contamination of the edible mushroom (33). Thus, while LiCl might be applied to prevent fruiting in some mushroom-forming fungi, it can also inhibit the contamination during manufacturing in some scenario. In addition to $\mathrm{LiCl}$, other agents that specifically target GSK-3, can also prevent the development of fruiting body. In support of this conclusion, CHIR99021 trihydrochloride, an alternative GSK-3 specific inhibitor that acts through a distinct mechanism, also inhibits fruiting body formation. Other known GSK-3 inhibitors include: Maleimide Derivatives; Staurosporine and Organometallic Inhibitors;

337 Indole Derivatives; Paullone Derivatives; Pyrazolamide Derivatives; Pyrimidine and

338 Furopyrimidine Derivatives; Oxadiazole Derivatives; Thiazole Derivatives; and Miscellaneous Heterocyclic Derivatives. $(18,34)$

341 The sensitive window to $\mathrm{LiCl}$ is from mycelium, hyphal knot, initial to stage-1 primordium.

342 This indicates that the $\mathrm{LiCl}$ may inhibit fruiting through affecting the cell differentiation.

343 Inhibitors of GSK-3 were shown to maintain the mouse and human embryonic stem (ES)

344 cells in undifferentiated status, while removing inhibitor promotes differentiation into 
345 multiple cell lineages (35). The potency maintaining function of GSK-3 may be related to

346 protein degradation. After phosphorylated by GSK-3, many substrates will then be targeted

347 by ubiquitination for proteasome-mediated degradation. Undifferentiated cells are

348 proliferative because GSK-3 activity is limited by persistent unfavorable growing condition

349 signals. The effectors of GSK-3, such as transcription factors, are less modified by

350 phosphorylation and ubiquitination, so their half-lives are prolonged to enhance

351 stem/precursor cell proliferation (36). This suggested GSK3 interferes fruiting by interfering

352 cell specification. Deeper studies are needed to discover the mechanism in detail. 


\section{Reference}

355 1. Jones M, Bhat T, Huynh T, Kandare E, Yuen R, Wang CH, et al. Waste-derived low-

356 cost mycelium composite construction materials with improved fire safety. Fire and Materials. 2018 Nov;42(7):816-25.

2. Nguyen PQ, Courchesne NMD, Duraj-Thatte A, Praveschotinunt P, Joshi NS.

Engineered Living Materials: Prospects and Challenges for Using Biological Systems to Direct the Assembly of Smart Materials. Vol. 30, Advanced Materials. 2018. p. 1704847.

4. Nguyen PQ, Courchesne NMD, Duraj-Thatte A, Praveschotinunt P, Joshi NS. to Direct the Assembly of Smart Materials. Vol. 30, Advanced Materials. 2018.

5. Grimm D, Wösten HAB. Mushroom cultivation in the circular economy. Vol. 102, Applied Microbiology and Biotechnology. 2018. p. 7795-803.

6. Appels FVW, Dijksterhuis J, Lukasiewicz CE, Jansen KMB, Wösten HAB, Krijgsheld Reports. 2018;8(1). practice. JOURNAL OF CLEANER PRODUCTION. 2018;186:570-84. 
375 8. Khamrai M, Banerjee SL, Kundu PP. A sustainable production method of mycelium biomass using an isolated fungal strain Phanerochaete chrysosporium (accession no: KY593186): Its exploitation in wound healing patch formation. Biocatalysis and Agricultural Biotechnology. 2018;16:548-57.

9. Jones M, Bhat T, Huynh T, Kandare E, Yuen R, Wang CH, et al. Waste-derived lowcost mycelium composite construction materials with improved fire safety. Fire and Materials. 2018;

10. Kilaru S, Hoegger PJ, Kües U. The laccase multi-gene family in Coprinopsis cinerea has seventeen different members that divide into two distinct subfamilies. Current Genetics. 2006 Jul 28;50(1):45-60.

11. Grimm D, Wösten HAB. Mushroom cultivation in the circular economy. Vol. 102, Applied Microbiology and Biotechnology. 2018. p. 7795-803.

12. Appels FVW, Camere S, Montalti M, Karana E, Jansen KMB, Dijksterhuis J, et al. Fabrication factors influencing mechanical, moisture- and water-related properties of mycelium-based composites. Materials \& Design. 2018;

13. Silverman J. Development and testing of mycelium-based composite materials for shoe sole applications. 2018;

14. Kües U, Subba S, Yu Y, Sen M. Regulation of fruiting body development in Coprinopsis cinerea. 2016;(July).

15. Haneef M, Ceseracciu L, Canale C, Bayer IS, Heredia-Guerrero JA, Athanassiou A. Advanced Materials From Fungal Mycelium: Fabrication and Tuning of Physical 
16. Kosti I, Mandel-Gutfreund Y, Glaser F, Horwitz B. Comparative analysis of fungal protein kinases and associated domains. BMC Genomics. 2010 Feb;11(1):133.

17. Wang C, Zhang S, Hou R, Zhao Z, Zheng Q, Xu Q, et al. Functional analysis of the kinome of the wheat scab fungus Fusarium graminearum. Howlett BJ, editor. PLoS pathogens. Public Library of Science; 2011 Dec;7(12):e1002460.

18. Takahashi-yanaga F. Activator or inhibitor? GSK-3 as a new drug target. Biochemical Pharmacology. Elsevier Inc.; 2013;86(2):191-9.

19. Andoh T, Hirata Y, Kikuchi A. Yeast glycogen synthase kinase 3 is involved in protein degradation in cooperation with Bul1, Bul2, and Rsp5. Molecular and cellular biology. 2000 Sep;20(18):6712-20.

20. Moore SF, van den Bosch MTJ, Hunter RW, Sakamoto K, Poole AW, Hers I. Dual regulation of glycogen synthase kinase $3(\mathrm{GSK} 3) \alpha / \beta$ by protein kinase $\mathrm{C}(\mathrm{PKC}) \alpha$ and Akt promotes thrombin-mediated integrin $\alpha \operatorname{IIb} \beta 3$ activation and granule secretion in platelets. The Journal of biological chemistry. 2013 Feb 8;288(6):3918-28.

21. Tataroğlu Ö, Lauinger L, Sancar G, Jakob K, Brunner M, Diernfellner ACR. Glycogen synthase kinase is a regulator of the circadian clock of Neurospora crassa. The Journal of biological chemistry. 2012 Oct 26;287(44):36936-43.

22. Mleczek M, Siwulski M, Rzymski P, Budzyńska S, Gąsecka M, Kalač P, et al. Cultivation of mushrooms for production of food biofortified with lithium. European Food Research and Technology. 2017;243(6):1097-104. 
23. De Assunão LS, Da Luz JMR, Da Silva MDCS, Vieira PAF, Bazzolli DMS, Vanetti MCD, et al. Enrichment of mushrooms: An interesting strategy for the acquisition of lithium. Food Chemistry. Elsevier; 2012 Sep 15;134(2):1123-7.

24. Zhang F, Phiel CJ, Spece L, Gurvich N, Klein PS. Inhibitory phosphorylation of glycogen synthase kinase-3 (GSK-3) in response to lithium. Evidence for autoregulation of GSK-3. The Journal of biological chemistry. American Society for Biochemistry and Molecular Biology; 2003 Aug 29;278(35):33067-77.

25. Stajich JE, Wilke SK, Ahrén D, Hang C, Birren BW, Borodovsky M, et al. Insights into evolution of multicellular fungi from the assembled chromosomes of the mushroom Coprinopsis cinerea ( Coprinus cinereus ).

26. Plaza D, Lin C-W, van der Velden NS, Aebi M, Künzler M, Dacks J, et al. Comparative transcriptomics of the model mushroom Coprinopsis cinerea reveals tissue-specific armories and a conserved circuitry for sexual development. BMC Genomics. BioMed Central; 2014;15(1):492.

27. Rao PS, Niederpruem DJ. Carbohydrate metabolism during morphogenesis of Coprinus lagopus (sensu Buller). Journal of bacteriology. 1969 Dec;100(3):1222-8.

28. Li L, Stoeckert CJ, Roos DS. OrthoMCL: identification of ortholog groups for eukaryotic genomes. Genome research. Cold Spring Harbor Laboratory Press; 2003 Sep 1;13(9):2178-89.

29. Tataroglu O. Role of Glycogen Synthase Kinase (GSK) in temperature compensation of the. $2011 ; 1-88$. 
30. Ninkovic J, Stigloher C, Lillesaar C, Bally-Cuif L. Gsk3beta/PKA and Gli1 regulate the maintenance of neural progenitors at the midbrain-hindbrain boundary in concert with E(Spl) factor activity. Development (Cambridge, England). 2008;135(18):313748.

31. Casas-Flores S, Rios-Momberg M, Rosales-Saavedra T, Martínez-Hernández P, Olmedo-Monfil V, Herrera-Estrella A. Cross Talk between a Fungal Blue-Light Perception System and the Cyclic AMP Signaling Pathway. Eukaryotic Cell. 2006 Mar;5(3):499-506.

32. Zanolli F, Magalhães R, Paula D, Carlos L, Barbosa B, Francisco H, et al. cAMP signaling pathway controls glycogen metabolism in Neurospora crassa by regulating the glycogen synthase gene expression and phosphorylation. Fungal Genetics and Biology. Elsevier Inc.; 2010;47(1):43-52.

33. H.G.Wildman. Lithium chloride as a selective inhibitor of Trichoderma species on soil isolation plates. Mycological Research. Elsevier; 1991 Dec 1;95(12):1364-8.

34. Selenica M-L, Jensen HS, Larsen AK, Pedersen ML, Helboe L, Leist M, et al. Efficacy of small-molecule glycogen synthase kinase-3 inhibitors in the postnatal rat model of tau hyperphosphorylation. British journal of pharmacology. Blackwell Publishing; 2007 Nov 12;152(6):959-79.

35. Kirby LA, Schott JT, Noble BL, Mendez DC, Caseley PS, Peterson SC, et al. Glycogen synthase kinase 3 (GSK3) inhibitor, SB-216763, promotes pluripotency in mouse embryonic stem cells. Cooney AJ, editor. PloS One. Public Library of Science; 2012 Jan;7(6):e39329. 

available under aCC-BY 4.0 International license.

460 36. Westermarck J. Regulation of transcription factor function by targeted protein

461 degradation: an overview focusing on p53, c-Myc, and c-Jun. Methods in molecular

462 biology (Clifton, NJ). 2010 Jan;647:31-6.

463

464 
$\mathrm{a}$

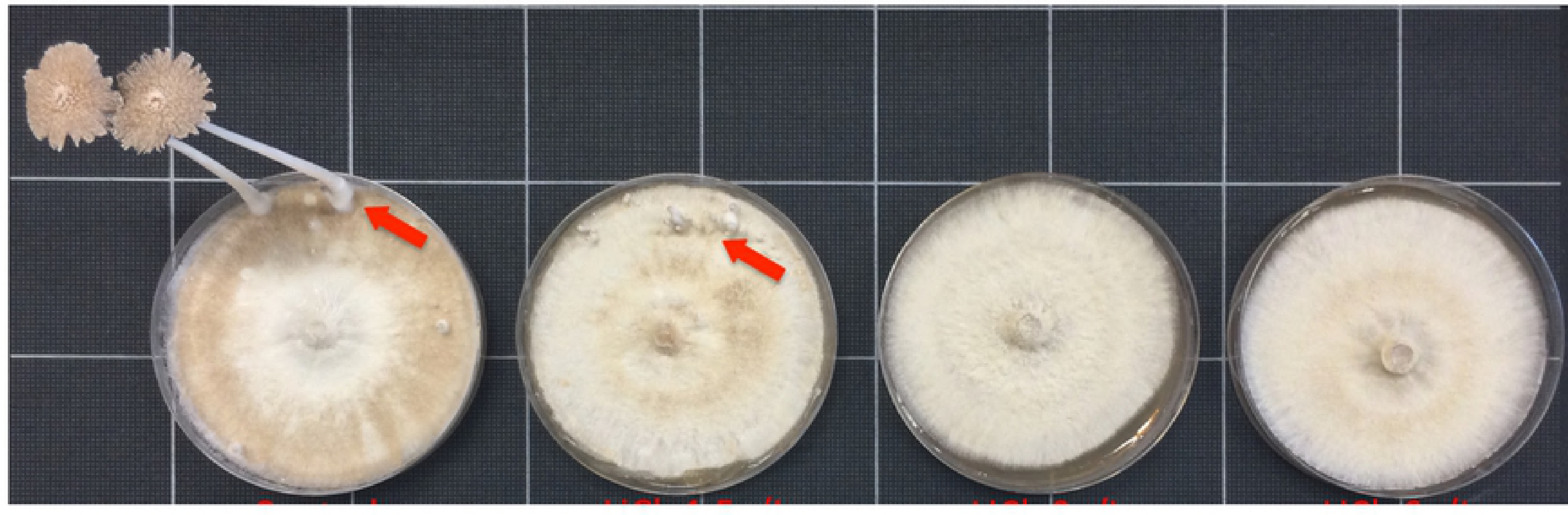

b
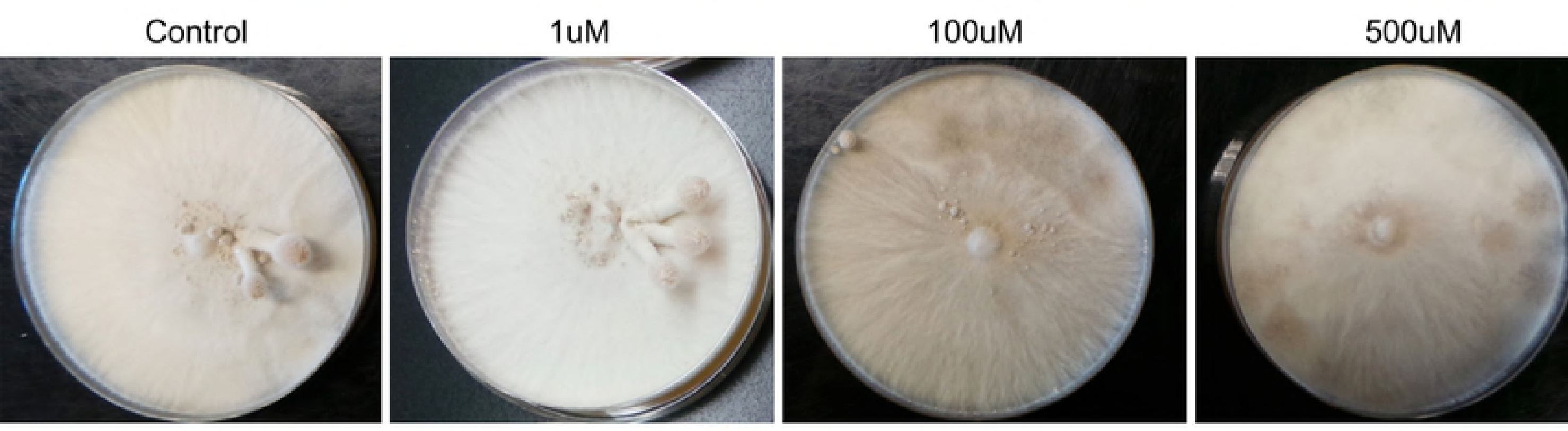

C

Control

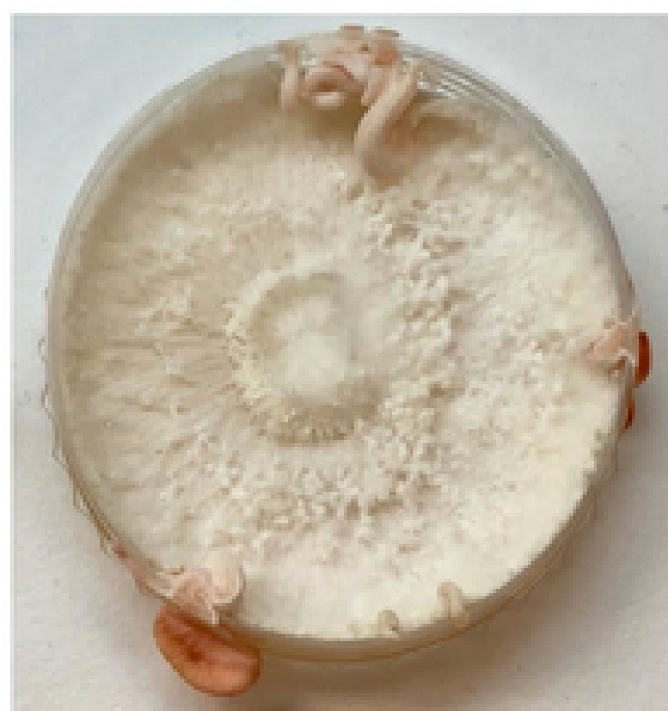

$2 \mathrm{~g} / \mathrm{L}$

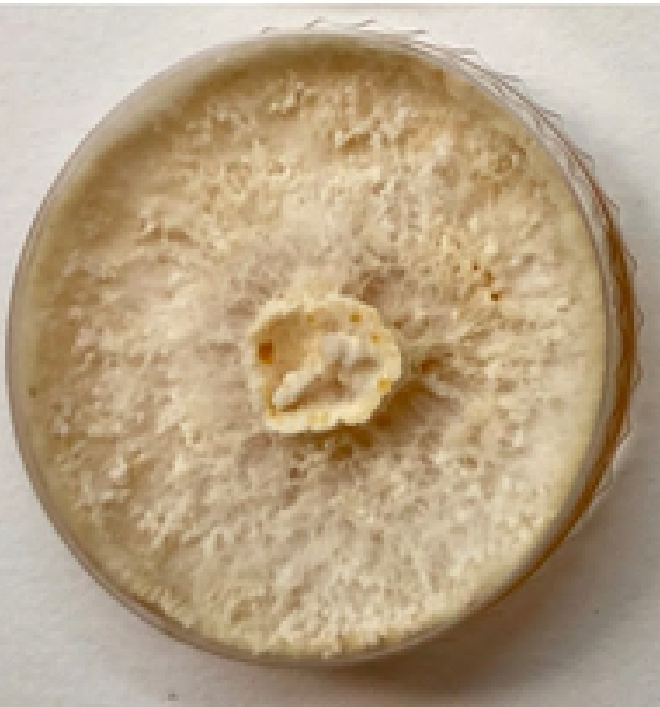

d
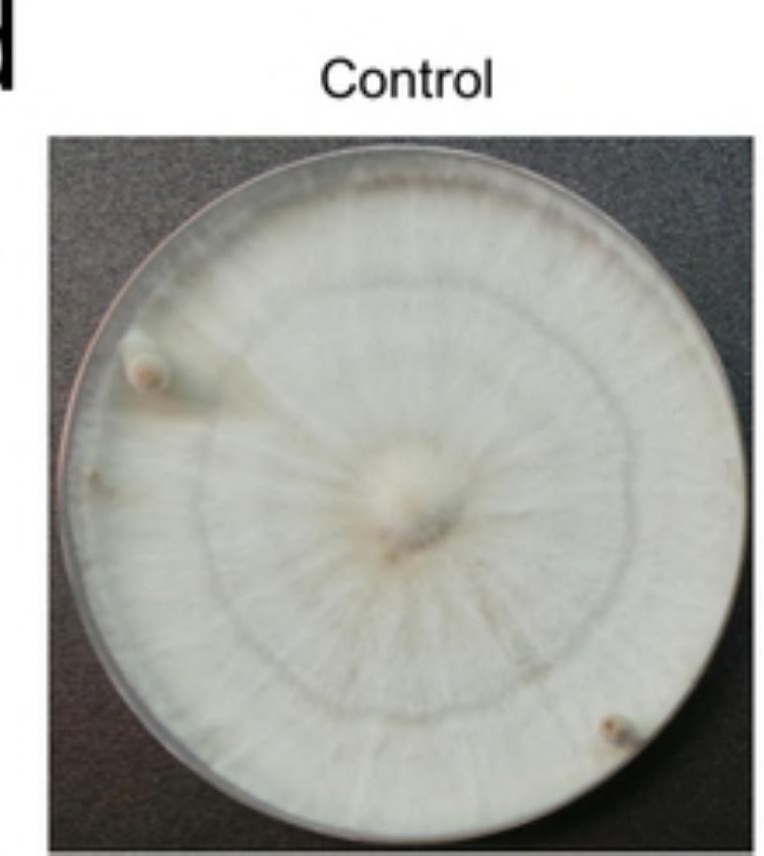

Cisplatin

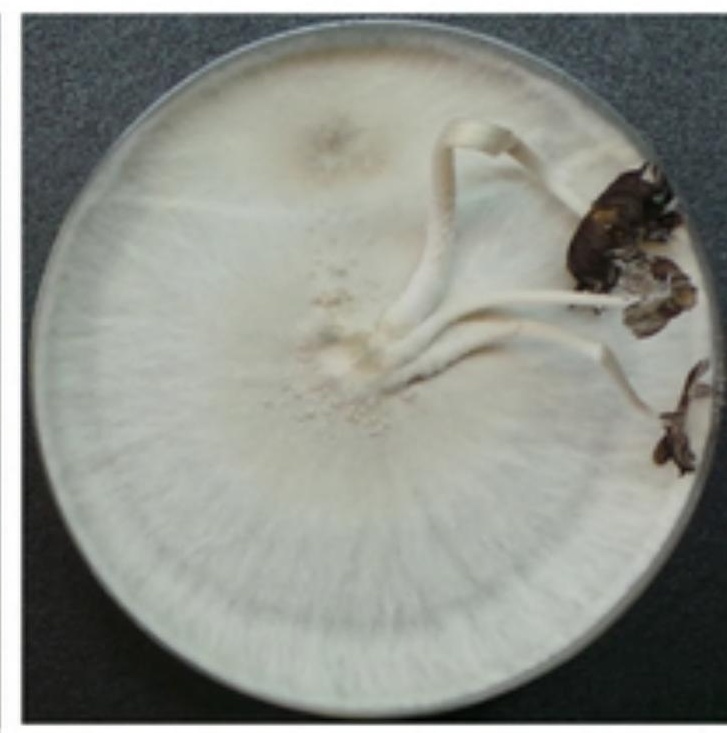




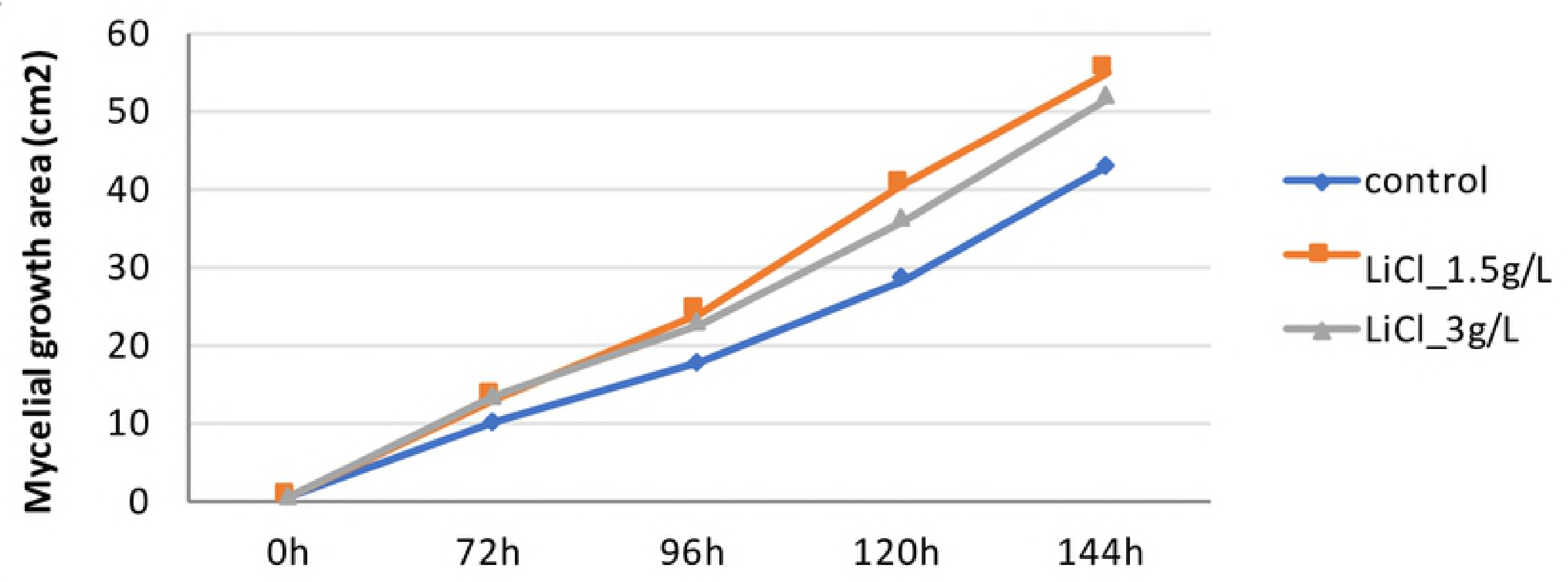

b

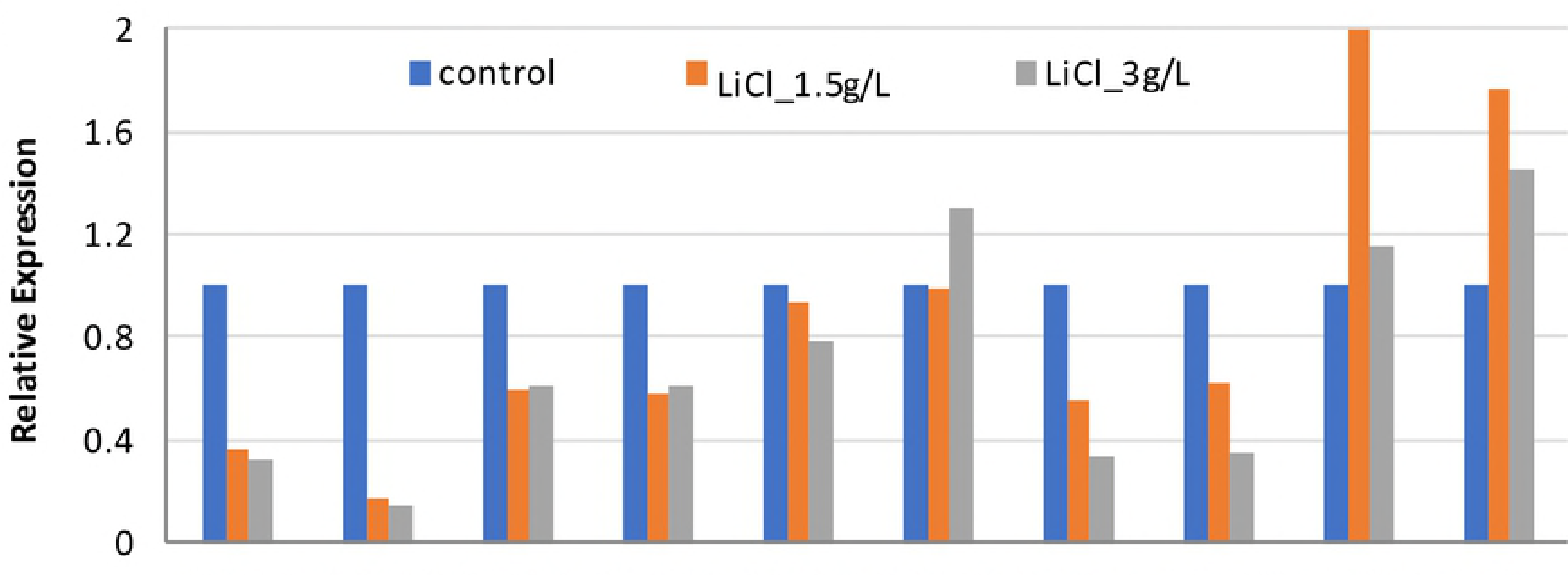
$1973-1$ 1973-2 3802-1 $3802-2$ 3881-1 $3881-2$ 5298-1 $5298-2$ 9429-1 9429-2 
Before adding LiCl
1 day after adding $\mathrm{LiCl}$
2 days after adding LiCl
3 days after adding LiCl
4 days after adding LiCl

\section{(a) Initial}
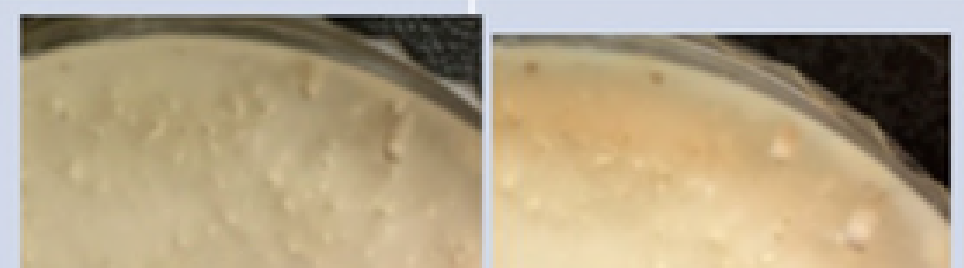

bioRxiv preprint doi: https://doi.org/10.1101/496265; this version posted December 13, 2018. The copyright holder for this preprint (which was not certified by peer review) is the author/funder, who has granted bioRxiv a license to display the preprint in perpetuity. It is made available under aCC-BY 4.0 International license.

(b) Pri 1
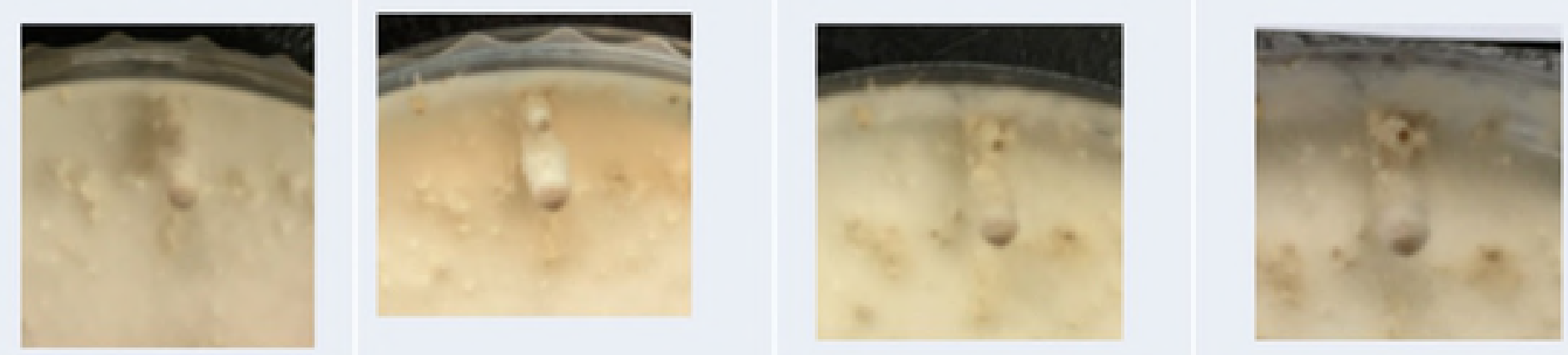

(c) Pri 2
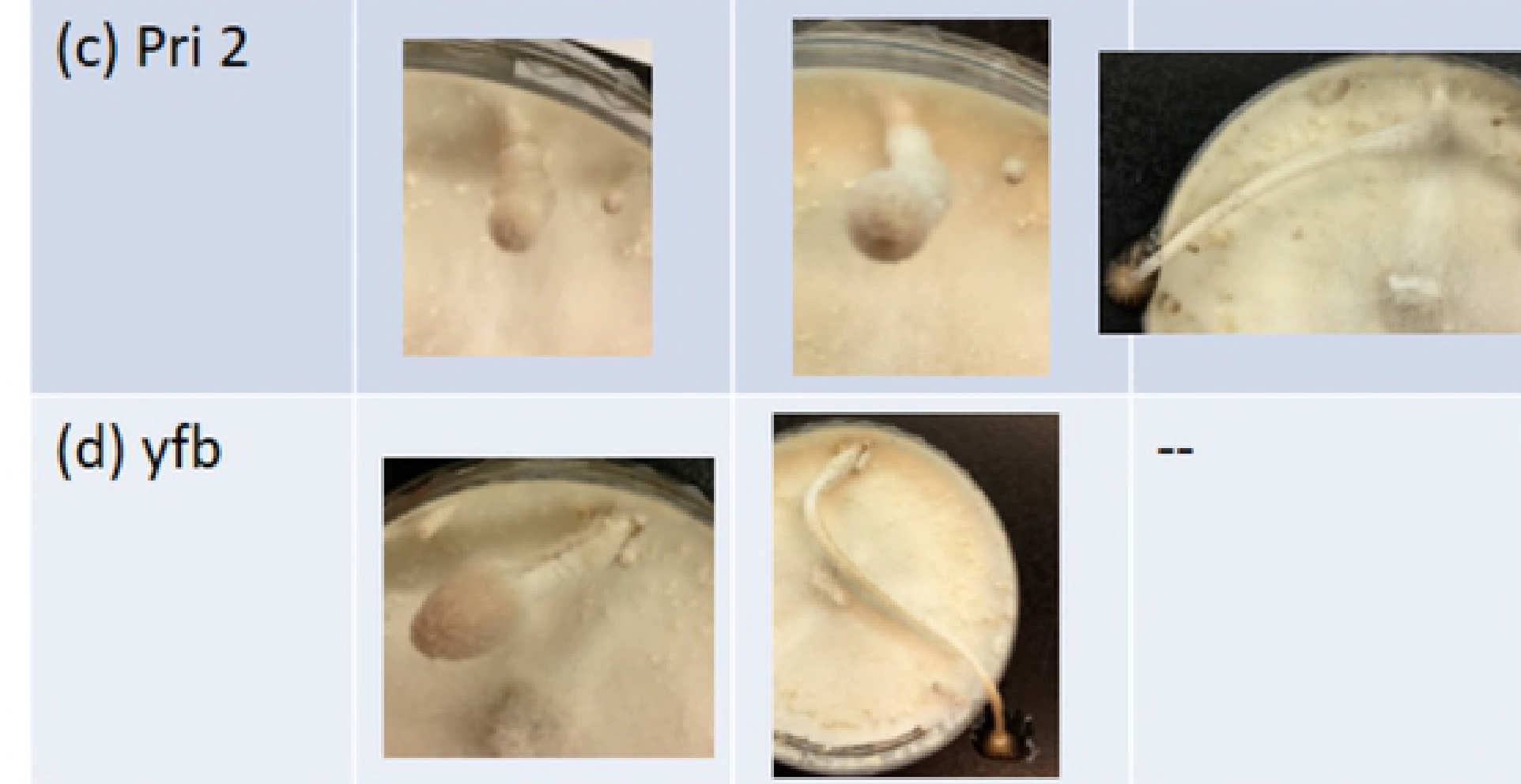

(d) yfb
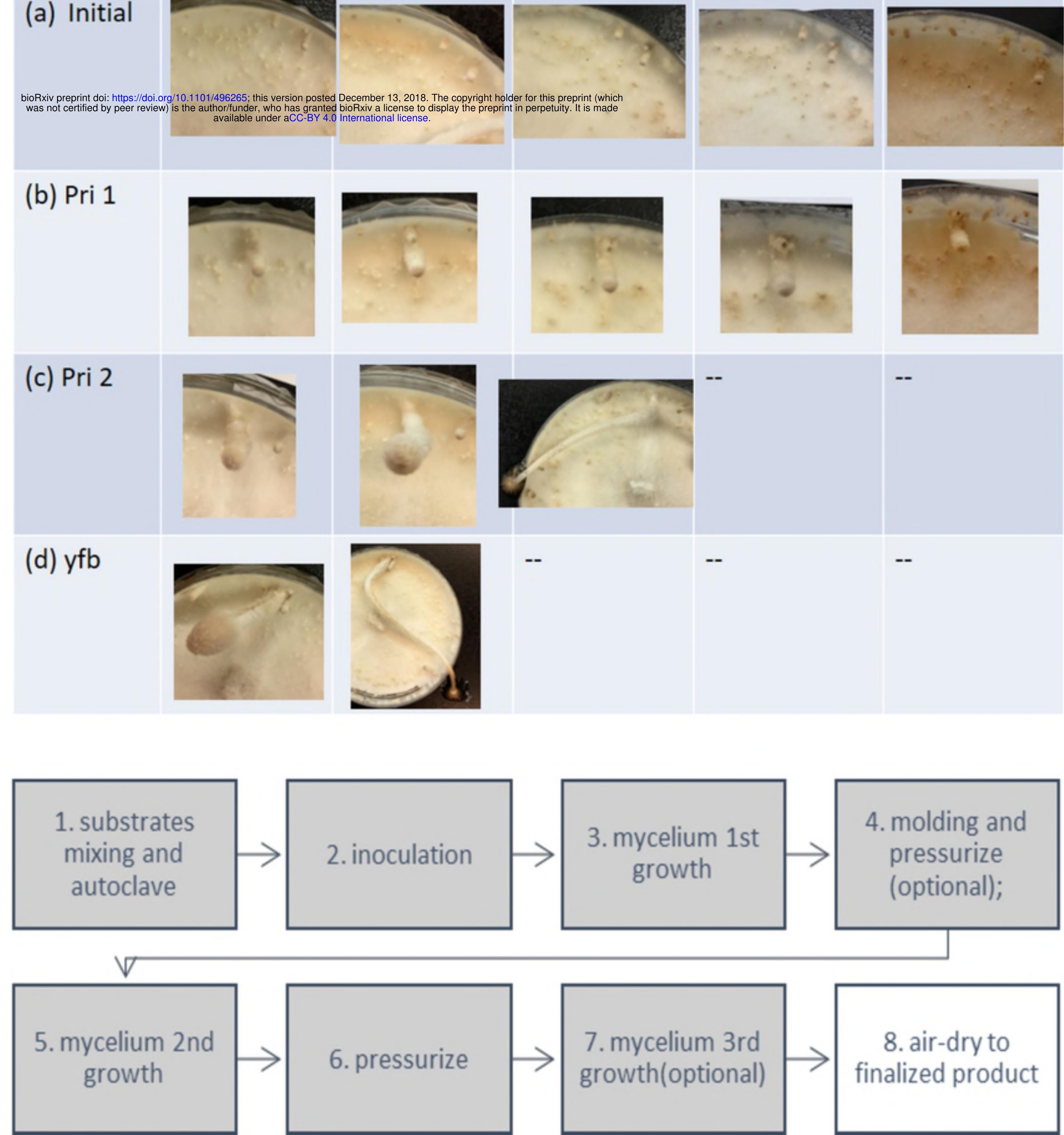
Favorable Nutrient

Glucose depletion

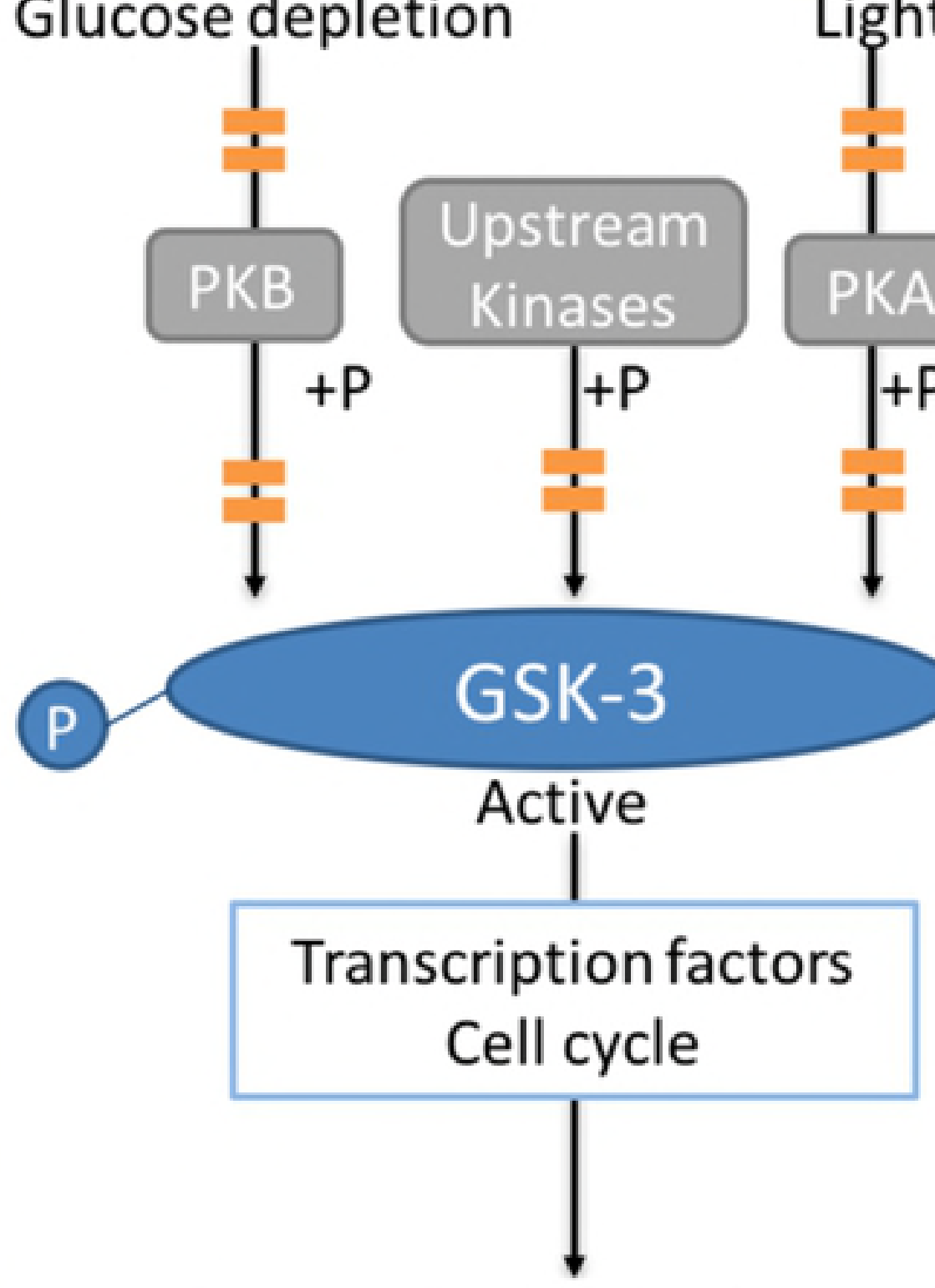

Fruiting Body Development
Unfavorable

Light

Light

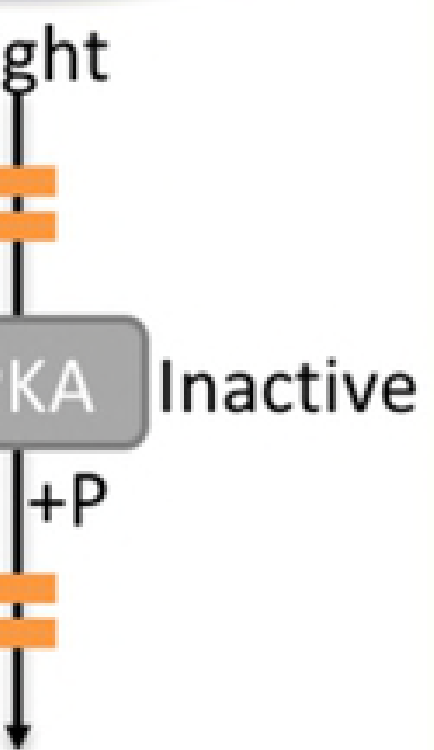

$+P$

更

Active

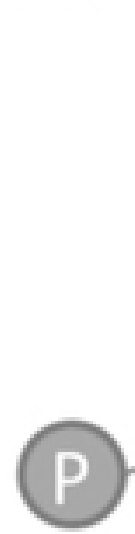

Nutrient

Glucose $\uparrow$

Light

Dark

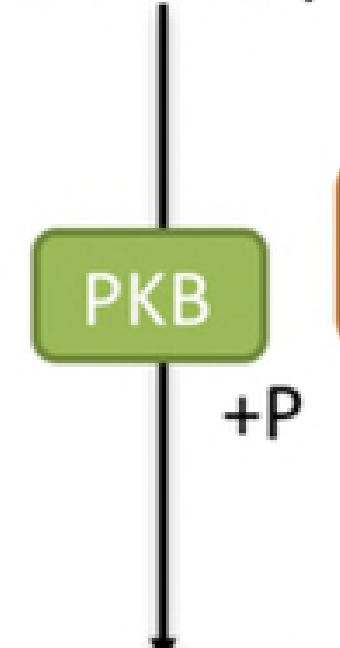

Upstream

Kinases

$+\mathrm{P}$

PKA $+\mathrm{P}$

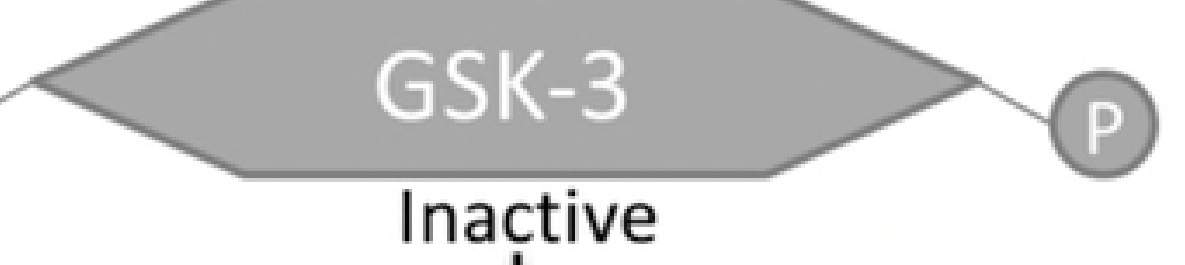

Transcription factors

Cell cycle

\section{No Fruiting Body}

